\title{
Philosophy Does Not Mean Love for Wisdom: Case study of Hebrew Old Testament Phalasaphiya, i.e., False-Prophecy, Produced Philosophy
}

\author{
Charles Ogundu Nnaji \\ University of Abuja, Abuja, Nigeria
}

\begin{abstract}
The study digs deep into the Semitic-Hebrew Old Testament roots of the word "philosophy" particularly, noting Judges 6:13, Hebrew "Niphilo-tha esh sophru-lanu-abbotenu” transliterated into koine Greek New Testament as "phi-lo-sophia” which means "wonder stories (or teachings) taught us by our fathers; with the figurative meaning as, ‘Are you sure God exists?’”. The study, through its Semitic textual linguistic methodology, also identified Deuteronomy 25:3, Hebrew "Ha-Philo ha-Shophet", which means "Human Judgments", is another form of the Hebrew "Phenosophi” (i.e., human additions, Deut 25:3); then, 2 Samuel 3:34, Hebrew "Na-Phala-yo-shophim” (i.e., outdated or dead); please see Colossians 2:8, or to fall and die;; also, 2 Kings 10:23, Hebrew “Phen-Yeo-Shophu” (i.e., Godless people seen also in Job 9:24, Hebrew “Phenoshophet” (i.e., Godless or blind Judgements = Note also Job 22:13, Hebrew “Ara-Phel-yi-shophet” = blind or bad judges; also in 2 Corinthians 4:4, Greek New Testament “Tuphlosen (i.e., blindness). The study observed that 1 Chro 2:47, Hebrew “Epha-we-shaaph” is the Noun form of the Verb "E-phala-shaaphi”, which means "cleverly invented, vague, obscure or added”. The study recommends more studies on the origins and true meaning of philosophy; particularly, looking at Genesis 50:1, Hebrew "Phiolyoseph" which means, “worthless and helpless human knowledge”, while Genesis 48:11, Hebrew "Philalyoseph means, "I thought (I will not see) you again (yoseph = Joseph). If we go to the Septuagint Greek Old Testament Genesis 37:3-4, the Greek “Phileo-Yoseph” means “(Israel), loved Joseph”. The same Septuagint transliterated Daniel 1:20, Hebrew "Haheretumim and "Ha (philah) shaphim”, as Sophoi and Philosophoi, i.e., magicians and wizards. The study has also included Hebrew O.T. words or dictionary which produced the word "philosophy".
\end{abstract}

Keywords: philosophy, love, wisdom, phalashapha, false, prophet

\section{Background to the Study}

Please note Hosea 7:1 Phalasaphqer; 2 Kings 10:23, Job 9:24, Hebrew "Phenishophet” (Falsehood) or Job 34:32, "Phalisipha” 2 Cor 4:4 Greek Tuphlosen; Psalm 58:2, Hebrew Tophilosun, etc. which means Manmade $=$ Col 2:8 Philosophy is empty human deceit. Thus, the search for the true origin of the word "philosophy" arose from our increasing knowledge of how to read and write the Hebrew Old Testament, where we met the Hebrew words "Philasapha, Baalsaphon, Phalashaphya, yoseph-phila, Phenoshophet, Ha-philoshophim”, etc.,

Charles Ogundu Nnaji, Ph.D., Department of Christian Studies and Religious Communications, Faculty of Arts, University of Abuja, Abuja, Nigeria. 
which lexically means, ancient human knowledge, and judgments obtained through occultic or magical means e.g., Daniel 1:20 and Daniel 2:2-4, noting Daniel 1:20 and (5:7), Hebrew "Ha-heretumim Veha-phila-shaphim" and "Laoashaphaya", transliterated in the septuaginta as "Sophoi and Philosophoi", which means sorcerers, magicians, and wizards (or enchanters; i.e., necromancy (Kittel, Friedrich, \& Bromiley, 1985). Seen also in Isaiah 29:13-14, Hebrew "Bi-phi-shophate" and "yosopher phila" then in Psalms 71:15 Hebrew "Phi-(li) Sepher”; also, in Psalm 50:16, Hebrew "Lasapher-Phiq”, i.e., to teach God's commandments (as magic) or, wrongly adding magic teachings to the word of God. This also takes us to proverbs 16:23, Hebrew "Phi-u-le-sepher", which means "think before you talk". Also, noting Job 34:32, Hebrew "Phaalisipha" translated as "stop committing evil, or deliberately committing evil", which is related to Psalms 58:2 “To-philosun”, transliterated and repeated in Colossians 2:8, Greek N.T. "Tes-philosophia” (i.e., human teachings and reasoning, Douglas, 1993, p. 701). We ought also to note Job 5:9 and 9:10, Hebrew "Ni-phila-ed-m-sepher" which was translated as "uncountable, inexplicable, and unsearchable" (Note Psalm 139:6 \& 14, Hebrew "Philiah-Philiah” = Job 37:16, Hebrew "Miphilashi-ab-m-shapher” [Gen 15:5] = i.e., wonders beyond man [Brown \& Smith, 2008; Harris, Archer, \& Watke, 1980; Brown, Driver, \& Briggs, 2014, etc.]). Deuteronomy 17:8 "Phila...m-shaphate” means difficult to judge, while transliterating Daniel 1:20 and Numbers 11:4 "Ha-saphsoph” = "(Haphilah-saphsuph)” or "Haphilashaphim”, gives us "godless people; enchanters or wizards = necromancy (Kittel et al., 1985, pp. 1269-1271).

\section{Objective and Focus of the Study}

The study, as stated in the abstract, focuses mainly on presenting Hebrew Old Testament words which clearly prove that philosophy does not mean love for wisdom. The Hebrew "Phi-u-losepher", i.e., to speak what your mind tells you, seen in Proverbs 16:23, Hosea 6:5, "Phi-l-sapher”, Psalm 40:14-15, and Isaiah 29:13-14, etc. simply means, "human” (BDB, 2014, p. 607). Te Egyptian "Phallu and Hebrew "Phaalu” means "man or male” genitals: noted in 1 Chronicles 5:1-3 and Deuteronomy 23:2, phallusphaq (Num 26:1-5-11), i.e., human’s who oppose God. The study has also produces a dictionary of Hebrew Old Testament words which produces the word, "Philosophy.

\section{Statement of Problem of the Study}

For at least, about, 100 years now, people deeply believe that "philosophy" is Greek, and that it indisputably means "love for wisdom", thus it becomes very difficult to believe new studies which clearly prove that philosophy does not mean love for wisdom; even the language transliteration of Revelations 9:11 confirms Hebrew O.T. Psalm 55:9, "Bla-Phalag-losona and Bla-(phla) sapha” of Gen 11:7, e.g., all these compared to Genesis 11:9 \& 11 means that when Hebrew O.T, "B" is transliterated to current English (Rev 9:11) "BLasapha” becomes "Phalasapha”, i.e., "to challenge God” clearly written as "phalsaphia”, in Job 34:32 and Judges, 6:13 (Num 26:1-5). Clearly, "Blashphemia is Philosophia” (Arabic "Falsafa” English "Falsify", means to destroy or to confuse. We also note that Baba is Papa, Abbot is Patriarch (i.e., father), Abad is Phagos i.e., to destroy, while the name "Shophac" in king James and NIV Bible translations, is "Shobac" in the Goodnews Bible translation (1 Chronicles 19:16) (please note that 2 Kings 3:11 and 12, Hebrew "Yehoshaphat" and 2 Chronicles 23:1, Hebrew "Elishaphat" (God judges) has its opposite as "philashapha", i.e., human Judgements, seen in Deuteronomy 25:2 and in Deuteronomy 17:8, Hebrew, "Philoshaphat”, i.e., Judges in Israel "Phila" is extraordinarily human, judge is "Shophet", while Hebrew "Sopher is "an understanding and 
learned scribe. Also, Zidon is Sidon, Zadok is Sadducee, while zakat is sadaqa or sediq (i.e., righteousness; also zina [or zoni] is sinner, etc., 1 Chronicle 27:32-34); the Hebrew "Sophe” is a watchman or Prophet (In Ezekiel 33:1-2 "Losophe", is to appoint a prophet; then "pha-l-m-sophe" is falsehood (Hosea 5:1, 1 Chronicles 27:32, Isaiah 56:10, Ezekiel 33:2, Ezekiel 3:17, and Jeremiah 6:17 [Harris et al., 1980; Mansoor, 2002; BDB, 2014; Brown \& Smith, 2008; SDHS, 2005]).

\section{Method of Study}

Consultations of Hebrew, Aramaic, Phoenician, and Assyro-Babylonia classist language texts produced this study's citations, such as Brown et al. (2014), Brown and Smith (2008), Metzger (2001), Mansoor (2002), Kittel et al., 1985, Newman et al. (1983), Nestle, 1971, Harris et al. (1980), Douglas (1993), and SDHS (2005), etc.

\section{Definition of Terms}

\section{Philosophy}

Newman et al. (1983, p. 196) defined "philosopher" as he who teaches a way of thinking (or thought) while New and Phillips (1953, pp. 94-961) called philosophy "to love knowledge"; then Robinson and Davidson (2007, p. 1040) described philosophy as searching for truth on the origins and nature of existence, i.e., through human reasoning, human reflections, and human judgements. Socrates (i.e., sorcery) was sentenced to death in 399 BC by leaders of Athens for being a member of the cult of Delphi called "baali" in Ecclesiastes (i.e., Eccl 12:11; BDB, 2014, pp. 80, 117-127). Philosophy is thus, an activity, or a quest after knowledge; it is an activity of human thought-patterns or wider ways of human thinking and judgements (Philosophy NET, 2019). Please note Isaiah 20:4, Hebrew "Yahphe-vi-he-supha (i.e., bare or empty) compared to Col 2:8, "philosophy".

\section{Love}

Simpson and Weiner (1989) and Eke (2001, p. 258) are of the view that, love simply means "to be fond of somebody or something”, to the extreme of sexuality, probably, or what you cannot do without. This brings in questions of addiction (Hebrew "Ahabah” or Aheb; BDB,: 2014, pp. 22-26, 525-532). We learn that the word "love” probably came from the Hebrew Old Testament. "Leui” or "Levi” (Gen 29:34 Number 3:11-13), which means that which belongs to me, or to be fond of or to be attached to something (Brown \& Smith, 2008, p. 47).

\section{Wisdom}

Eke (2001, p. 421) defined wisdom as being wise confirmed by Robinson and Davidson (2007, pp. 1628-1629) that wisdom means "to know, learned and sensible: The word wisdom is from, "to be wise, prudent and sensible: Pfeiffer and Harrison (1953, pp. 1053, 1814-1815 in matters of love for wisdom cited the Greek "Phileo and Agapao", to mean physical love for (philia) and Divine Love (for Agapao), was it can be sexual love, i.e., erotica from BDB (2014, pp. 16-28); those three cited Greek words for love, i.e., phileo, Agape and Eros were actually transliterated from the Hebrew, "Eluphi”, Agabah (or Aheb = Aphili) and Eri:(Genesis 38:7, Eke, 2001, pp. 258, 421) also noted that the Igbo word for love is "Ihunanya", while wisdom is "amamihe" or Uche (i.e., to know) cognate "Yoruba”, “Ogbon” (i.e., sensible) contrasting senselessness.

\section{Phalashaphiya and False-Prophets}

In ancient Israel, false-prophets were called "Phalishophi, Phalasaqer, or phaalisaphqer (i.e., deceit Hos 
7:1) or Niphalasophou”, i.e., dead or outdated messages = 2 Samuel 3:3-4 (please see Wycliffe commentary on philosophy). A false-prophet is one who falsely claims the gift of prophecy or divine inspiration, or who uses that gift for evil ends. Often, someone who is considered a "true Prophet" by some people is simultaneously considered a "false-prophet" by others, even within the same religion as the "prophet" in question (False-Prophet NET, 2019). Also, Pfeiffer, Vos, and Rea (2003, pp. 1336-1337; Wycliffe; Bible Dictionary Hendrickson; Massachussets) they cited Colossians 2:3 "Philosophia”, as heretic Jewish ascetic teachings seen in Hosea 7:1 as Hebrew "Phaalisaphqer or Falsehood Also noted in Job 34:32-Phaalisipha "(i.e., deliberate evil = Psalm 58:2-3 “Tophilosun”) (BDB, 2014; Brown \& Smith, 2008).

\section{Comparative Blasapha, Philasapha and Philosophia}

This section is intended to show that "Blasphemia is philosophia".

Newman et al. (1983, p. 196) defined philosophia as "a teacher of a way of thinking, (or thought) which corresponds to New and Philips (1953, pp. 116-117) who described the Socratic school as teaching people how to arrive at faultless judgements through systematic or crafty reasoning; Kittel et al., 1985, p. 1269) stated that people do not really know the origin of the Greek word "Phil", this which agrees with Metzger (2001), that the word "Philosophy" has not appeared in Greek lexicons; however, note the Hebrew Old Testament Psalm 55:9 "Bla-phalag Losona” compared to Genesis 11:7 and 9, "Bla-sapha”; not forgetting Genesis 6:4, Hebrew "Ne-Philim (i.e., Giants) or "Baal” and “Ophl” which means "Giant, hill, master (or Rabbi) or highly placed. This is also found in 1 Chronicles 19:16 "Shophach (KJV \& NIV) or Shobach (Goodnews Bible, 2008) noting that the word "Baba" is from "Baal” (or patriarch) which means "Husband, father or a godless leader"; also found in Ecclesiastes 12:11 "Baali-Sohophot" transliterated as "Phalishopha” i.e., ancient sages, Judges, masters and assembly speakers (Douglas, 1993, p. 1419). The phonecian "Baali” gave us the "Nephilim” of Genesis 6:4, which implies ancient godless masters of secret assemblies, or of the occult; also called "La-Ashaphiya" in Daniel 2:27, sorcerers (i.e., Socrates) or Magicians, seen in Daniel 1:20 as "Ha-phila-ashaphim" which means that, in ancient times, people like Socrates (i.e., sorcerers of Delphi; Asaju (1999, p. 36) went to cultic places, to find out, or resolve problems through magic (i.e., sorcery: Deut 18:1-14; Num 23:14, I-end, Num 24:1; the baali-shophim or zophim; note that “zion” was originally “sion” or Sinai).

\section{Research Observation and Finding (Pythagoras did not Coin Philosophy)}

The study observed that, till date, no one seems to know the true origins and true meaning of the word philosophy; Metzger (2001, p. 3) cited over fifty Greek Lexicons, all which do not have the word "philosophy”, but they have the word "Sophron" which means "understanding". On the contrary, the Hebrew Old Testament has the Hebrew "Ha-Philasophim”, i.e., human judgement and understanding more than fifty times in various forms, such as Deuteronomy 17:8 "Phila-m-shaphat", difficult to judge, then Jeremiah 6:26 "Philashapher", which means "Earthly", etc. (Brown \& Smith, 1990, p. 798). Pythagoras did not coin the word philosophy; if he did, let us have the proofs: Note Deut 25:1-3, "Ha-philoshophet”, i.e., human judgement; Deut 17:8; Job 22:13 and 2 Chro 23:1-Yehoshaphat, or Elishaphat = God judges (opposite "Philashophet" = human judgment, etc., BDB, 2014, pp. 44-46). Please note 1 Chronicles 2:47 "Ephaveshaaph" is noun, while its verb is "Niphilashaapha”, i.e., cleverly added; or “Eliasaph” (Num 3:24, God adds; Gen 30:24, Opposeite: Phenisophia $=$ Human additions $=$ Deut 25:3 do not add more). 


\section{Research Recommendations}

The research study recommends more, objective, research investigations into the true roots and true meaning of the word "philosophy".

\section{Research Contribution (i.e., Hebrew O.T. Words Which Produced Philosophy)}

This study has included over 100 Hebrew Old Testament words which helps this study to etymologically prove that the Greek "Philosophia" was indisputably transliterated from the Hebrew Old Testament "Philosophet" (ancient Israelite Judges, Deuteronomy 25:1-3 or Phaalisipha-cognate phalashaya = Arabic "Falsafa" = English "Falsify", etc. Hos 7:1, 1 Chro 2:47 = Ephah-la-shaap phalashaphiya falsehood or human additions).

Table 1

Mini-Dictionary of Hebrew Old Testament (Aramaic) Words Which Produced the Word "Philosophy"

\begin{tabular}{|c|c|c|c|}
\hline & Hebrew & Greek & English \\
\hline 1 & Phallu: Num 26:1-5; “Phallusphaha” & Philos & $\begin{array}{l}\text { Phallic (i.e., male organ) or Human Opposition } \\
\text { to Num 26:1-11 }\end{array}$ \\
\hline 2 & $\begin{array}{l}\text { Leviticus 5:4, Isaiah 29:13-14, } 1 \text { Corinthians } \\
\text { 1:18-20, "Phir-Ba-Lo-Sophate". } \\
\text { Yephtha or Naphtal.0 } \\
\text { Sophi }\end{array}$ & $\begin{array}{l}\text { Kenophos = Jer 46:19-20 } \\
\text { Memphis (Heb “Nophil)=Egypt } \\
1 \text { Chronicles 2:47-Caleb’s } \\
\text { concubines; Philegs. Also see } \\
\text { "Ephelashaaph” = obscure desires, } \\
\text { human excesses. } \\
\end{array}$ & $\begin{array}{l}\text { Philosophia = Human speech and human } \\
\text { teachings. } \\
\text { Empty, or barren, Colossians 2:8, Col 2:4 = } \\
\text { persuasive. }\end{array}$ \\
\hline 3 & $\begin{array}{l}\text { a. Balu, Phalu, Philah, Phileg } \\
\text { Soephim } \\
\text { Phallusphaha Numbers 26:5 (rebely) } \\
\text { Blasphama and Philasphama } \\
\text { Nophili (i.e., Egypt) or deceit: Amos } \\
\text { 8:5-Epha-(KJV) Menophis, (Hos 9:6) Jeremiah } \\
\text { 46:19-Memphis-Egypt Nophi-l-ashama worthless } \\
\text { or earthly; Jer 6:26, "Philashaphar; (Earthly) } \\
\text { b. Job 16:13-Phala-Shopeq" }\end{array}$ & $\begin{array}{l}\text { Jer 46:19 (KJV) Greek “Memphis } \\
\text { from Hebrew “Menophis” root } \\
\text { “Nophili” (Hosea 9:6, Isaiah 19:13 } \\
\text { (KJV) }\end{array}$ & $\begin{array}{l}\text { a. Confuse, divide, Psalm 55:9 \& } 10 . \\
\text { Divide, limp in-between. } \\
\text { Double minded, } 1 \text { Samuel 18:21 or rebel family, } \\
\text { against God. } \\
\text { b. To pierce and Spill (= to kill) }\end{array}$ \\
\hline 4 & $\begin{array}{l}\text { Phallu-Sophaqu, Isaiah 2:6, Num 26:5-11 (foreign, } \\
\text { adulteration; Ezk 23:45). } \\
\text { Jer 6:26-Philashipher (Earthly) }\end{array}$ & $\begin{array}{l}\text { James 3:15-17 “Phaulos-Sophia” } \\
\text { (Earthly Wisdom) }\end{array}$ & $\begin{array}{l}\text { To oppose or accuse, Psalm 50:8. } \\
\text { Egypt is pagan or ungodly or wasted. Jer } 46: 19 \text {, } \\
\text { Isaiah 19:13. } \\
\text { Pagan; phaqu. }\end{array}$ \\
\hline 5 & 1 Chronicles 2:47 Ephahlashaaph & & $\begin{array}{l}\text { Adultery, adulterated. } \\
\text { Obscure, dark or bad human desires, or } \\
\text { excesses. }\end{array}$ \\
\hline 6 & $\begin{array}{l}\text { Ephah and Shaaph (1 Chro 2:47) = cleverly } \\
\text { invented } 2 \text { Peter 1:16 (Greek "Sophismenos) }\end{array}$ & & To lead astray or to deceive (desires). \\
\hline 7 & $\begin{array}{l}\text { Hebrew “Ephalashaaphi” } 1 \text { Chro 4:47(obscure and } \\
\text { misleading) } 1 \text { Chro 19:16, “Shophach”-(pagan } \\
\text { leader). }\end{array}$ & & $\begin{array}{l}\text { To deceive (Hebrew “Yephthah”), or to be } \\
\text { deceived (i.e., philosophota). }\end{array}$ \\
\hline 8 & $\begin{array}{l}\text { Philasophatic (Phoenician "Basophatayu”, } \\
\text { Revelations 9:11, Abad is Apol; Balm is Rapha, } \\
\text { etc.). }\end{array}$ & & $\begin{array}{l}\text { Spoken with the human mouth. Leviticus 5:4, } \\
\text { Isaiah 29:13-14.; Psalm 71:15, Eccl 10:12 }\end{array}$ \\
\hline 9 & $\begin{array}{l}\text { Hebrew "Yephtha” and Naphthali or Naphthalsapha } \\
\text { (Genesis 49:21, etc.). }\end{array}$ & $\begin{array}{l}\text { Phttah; ophtalmos (to open-eyes) } \\
\text { Mark 7:34 “Hebrew } \\
\text { "Ephathah”-open. } \\
\end{array}$ & $\begin{array}{l}\text { Phatasophat (open cophes your lips and speak) } \\
\text { flattery. }\end{array}$ \\
\hline 10 & Phili, Philisti from Phalasu or Palasti = Nephu. & philistinos & Adulterated, adulterate, or idolatry \\
\hline 11 & Philu & & To find out by casting lots \\
\hline 12 & Judges 6:13 “Niphilasophru” & & Wonder ancient teachings. \\
\hline 13 & Pha-la-sophiqu-(paqu) (Isaiah 2:6) & & To fall, pagan, (foreign) or a trap. \\
\hline 14 & BDB, 2007, p. 250 Shephelah & & Lowliness, lowland \\
\hline 15 & $\begin{array}{l}\text { Phaqid-phicol (overseer, Nehemiah 12:42). Gen } \\
\text { 21:22 }\end{array}$ & commander & Leader, king. \\
\hline 16 & $\begin{array}{l}\text { Phalasaphaq (pagan-leader-philasa, i.e., pileser-2 } \\
\text { Kings 16:7 \& 10). }\end{array}$ & & To mislead or lead astray, Isaiah 2:6. \\
\hline
\end{tabular}


(Table 1 continued)

\begin{tabular}{|c|c|c|c|}
\hline & Hebrew & Greek & English \\
\hline 17 & Genesis 21:22, phicol-pagan leader & & $\begin{array}{l}\text { Philosaophaqu = Philisti: Deceit, see Colossians } \\
\text { 2:8 Philosoaphia, Psalm 40:15, to lead astray, to } \\
\text { kill. }\end{array}$ \\
\hline 18 & $\begin{array}{l}\text { Aphal-sophia (Numbers 14:14, Aphiler; BDB, } \\
\text { 2007, p. 779) }\end{array}$ & & Heedless, rejects advice (i-too-know) \\
\hline 19 & Phallu & & Phallic-penis; uncircumcised, Col 2:8-13 \\
\hline 20 & $\begin{array}{l}\text { Phalusphaha (male cult), rebellion. Numbers } \\
\text { 26:5-11. }\end{array}$ & & Manhood \\
\hline 21 & Nephel (mound) or Ophel (Hill) & & Pregnancy/abortion \\
\hline 22 & Miphiloseab & & Floating, balancing \\
\hline 23 & Phelon. Daniel herd the Phelon = Holy One. & & Unknown Holy one: the extraordinary \\
\hline 24 & $\begin{array}{l}\text { Phalasu, fallacy (Naphulishaphat) Adultery: Ezk } \\
\text { 23:45. }\end{array}$ & & $\begin{array}{l}\text { Idolatry, polytheist following many opinions, } \\
\text { customs, and traditions. }\end{array}$ \\
\hline 25 & Philasu/ Philasti & & Senseless, worthless \\
\hline 26 & Jeremiah 6:26, Philastiaphar & & Roll in the dust \\
\hline 27 & Judges 16:23 “Philistisophu” & & Pagan gathering or pagan kings. \\
\hline 28 & $\begin{array}{l}\text { Sapham (2 Samuel 19:25, Micah 3:7, Ezekiel 24:17 } \\
\text { (BDB, 2007, p. 974). }\end{array}$ & & Moustache, irrelevant \\
\hline 29 & $\begin{array}{l}\text { Deuteronomy 15:8 “Tapheta” (Mark 7:34 } \\
\text { “Ephphathah) Arabic “Tafsir”, Isaiah 28:7 (16:3) } \\
\text { Phililim or Judges (909, pp. 811-814) Tophilah } \\
\text { (prayer). }\end{array}$ & & $\begin{array}{l}\text { Priests and interpreters } \\
\text { Note: priests and prophets were ancient judges } \\
\text { and interpreters Deuteronomy 17:8-11 } \\
\text { (19:15-19). }\end{array}$ \\
\hline 30 & $\begin{array}{l}\text { Book of Judges is Hebrew "Shophetim" (i.e., } \\
\text { shepherds), i.e., Hebrew name of Book of Judges). }\end{array}$ & Basileus & $\begin{array}{l}\text { Judges, leaders or Jewish kings. Shepherds, } \\
\text { leaders (Amplifier, 1987, p. 368). }\end{array}$ \\
\hline 31 & $\begin{array}{l}1 \text { Chronicles 19:16 “Shophach” (Gentile leader or } \\
\text { commander). }\end{array}$ & & Pagan leader \\
\hline 32 & Genesis 21:22 “Phicol” (leader) & & Leader \\
\hline 33 & Joshua 12:20 “Acshaph” (pagan kings) & Poimaino & Shepherd king. \\
\hline 34 & $\begin{array}{l}\text { Joshua 12:23 Naphoth-Ophel (Nehemiah 11:21) } \\
\text { Philil }\end{array}$ & & Height, hill, overseer, king. \\
\hline 35 & Yasaph (Joseph) & & $\begin{array}{l}\text { Exodus 30:22-24, Yoseph-Losoph-adding or } \\
\text { removing. Philosophy-human addition. }\end{array}$ \\
\hline 36 & $\begin{array}{l}\text { Ecclesiastes 3:14 “Lohsipha” Deuteronomy 4:2. } \\
\text { Philosophia. }\end{array}$ & & Philosopher-human additions. Human excesses. \\
\hline 37 & $\begin{array}{l}\text { Joshua 9:1 Phalshephelah (Phoenician } \\
\text { "Baalshaphela") }\end{array}$ & & $\begin{array}{l}\text { Low, lowly, lowlands, valley, worthless } \\
\text { (Jeremiah 6:26). }\end{array}$ \\
\hline 38 & $\begin{array}{l}\text { Joshua 9:2 “Pheh” (Phonen) Genesis 41:44-45, } \\
\text { Egypt-Aramaiti, “Zaphenath Paneah” } \\
\text { (Phanea-Phone) Greek "Phonesophonias" }\end{array}$ & & With one voice or one accord (KJV). \\
\hline 39 & Greek "Phanesophonias" & & $\begin{array}{l}\text { The god (Sophoni) speaks (phanea) Hebrew Old } \\
\text { Testament, 2005, BDB } 2007 .\end{array}$ \\
\hline 40 & Morphet (oth) & & Divine wonder, miracle, Douglas, 1993. \\
\hline 41 & $\begin{array}{l}\text { Sepheq, Saphaq-Sophic (2 Peter 1:16) Job 20:22, } 1 \\
\text { Kings 20:10. }\end{array}$ & & Plenty, many cleverly invented stories. \\
\hline 42 & Saphsaph 1 Kings 20:10 & & $\begin{array}{l}\text { Earthly things, more and plenty dusts of } \\
\text { Samaria. Human opinions. }\end{array}$ \\
\hline 43 & Sophiqu-sophical-Isaiah 2:6-Philistisophiqu & & $\begin{array}{l}\text { Pagan, man-made gods, foreign ideas: Acts } \\
17: 18-22 .\end{array}$ \\
\hline 44 & Naphuphuli-Shaphate (Ezekiel 23:45) & & Adulterate from adultery \\
\hline 45 & Asaph (Asab) sophoth, sopha, sophatha & Ecclesia & $\begin{array}{l}\text { Assembly-Ephemeral, peripheral, river-bank, or } \\
\text { not deep enough. } \\
\text { (Note: Deep is Emeq = Amorq). }\end{array}$ \\
\hline 46 & Amos 8:5 (Zechariah 5:5-11) Ephah. & & $\begin{array}{l}\text { Symbol of deceit or to falsify; or obscure and } \\
\text { cleverly devised; 2Peter 1:16. }\end{array}$ \\
\hline 47 & Sapha, Sephi, Sophi, Numbers 23:3, Colossians 2:8. & & $\begin{array}{l}\text { Barren, empty (Zophon) empty or bare hill, } \\
\text { watch tower (sophi). }\end{array}$ \\
\hline 48 & $\begin{array}{l}\text { Shophet (Topheth-Sophet) Jeremiah 19:5-6, } \\
\text { Seraphin-Serphim = to burn, also fire } \\
\text { serpent-Numbers 21:1-9. }\end{array}$ & & $\begin{array}{l}\text { Fire divination (2 Kings 17:20-32-Sophara, } \\
\text { Sepharvaim-fire divination }\end{array}$ \\
\hline
\end{tabular}


(Table 1 continued)

\begin{tabular}{|c|c|c|c|}
\hline & Hebrew & Greek & English \\
\hline 49 & $\begin{array}{l}\text { Shophac (losophac) yum-suph or suphal-Red Sea. } \\
\text { Also ocean torrents and waves. }\end{array}$ & & $\begin{array}{l}\text { To Pour out male semen or fornication. } \\
\text { Colossians 2:8-13-uncircumcised. } \\
\text { Hebrew Old Testament, 2005, BDB 2007, p. } \\
1046 .\end{array}$ \\
\hline 50 & Saph & & Temple door \\
\hline 51 & Shaphah (Exodus 1:15) Shaphrah (mad woman). & & Maid, handmaid. \\
\hline 52 & Shaphir (Micah 1:10-11) & & Beauty, shining (sahar). \\
\hline 53 & Phalashaphiah, Greek Pallas Athene. & & Greek goddess of wisdom. \\
\hline 54 & $\begin{array}{l}\text { Cephel (BDB, 2007, p. 1049) cephelhakham-double } \\
\text { wisdom. }\end{array}$ & & Double \\
\hline 55 & $\begin{array}{l}\text { Shephecah Deuteronomy 23:2 (BDB, 2007, p. } \\
\text { 1050). }\end{array}$ & & Male organ \\
\hline 56 & Phallu (Egyptian) & & Male organ = Phili = human \\
\hline 57 & $\begin{array}{l}\text { Herephili = salvation through circumcision is } \\
\text { heresy: Col 2:8-13. Exd 4:26 (to spare = sophare or } \\
\text { heal = Rapha; through circumcision) Col 2:8-13. }\end{array}$ & & $\begin{array}{l}\text { Redeemed by circumcision, Colossians 2:8-13 } \\
\text { (A falsehood entering early Christianity). }\end{array}$ \\
\hline 58 & Shophiphion, or Pheten-Peten-python. & & Serpent: Genesis 49:17. \\
\hline 59 & Supheq-sophic & & The serpent attacks, Genesis 3:15. \\
\hline 60 & $\begin{array}{l}\text { 2 Samuel 17:23 “Ahithophel”. } \\
\text { Shaphan (1050). } \\
\text { Sophe-watchman-prophet: Ezekiel 3:17, Jeremiah } \\
6: 17 \\
\text { Sapphaire. } \\
\end{array}$ & & $\begin{array}{l}\text { Bad (Thophel: Jeremiah 19:5-6-Tophet) Brother } \\
\text { (ahi). }\end{array}$ \\
\hline 61 & $\begin{array}{l}\text { Nephel } \\
\text { shaphia (Beauty) } \\
\text { Saphia (not from God, Job 14:19, } 1 \text { Kings 19:20). } \\
\end{array}$ & & $\begin{array}{l}\text { Abortion (abad = Revelation 9:11, to } \\
\text { destroy-Apol). Job 16:13 “Phalashophac" (to } \\
\text { destroy) }\end{array}$ \\
\hline 62 & Sophaoth (hidden, witchcraft; Ezekiel 13:18-20). & & sophen-serpent, witchcraft. \\
\hline 63 & Shophat Ezekiel 23:45 & & Ancient human customs \\
\hline 64 & Aphar (Aphra = Afriq), BDB, 2007, pp. 749-750. & & $\begin{array}{l}\text { Dust, worthless (Also mountain god). Jer 6:26, } \\
\text { Micah 1:10-11. }\end{array}$ \\
\hline 65 & $\begin{array}{l}\text { Soph or Suph (the end) to kill-suffocate (ibid 692), } \\
\text { Ecclesiastes 12:13, Esther 9:28, Isaiah 66:17, } \\
\text { Jeremiah 8:13, Zephaniah 1:2. }\end{array}$ & & Conclusion, end of the matter. \\
\hline 66 & $\begin{array}{l}\text { Psalm 104:18 (Ezekiel 8:1-12) Shaphani } \\
\text { Ph-loshaphan }\end{array}$ & & $\begin{array}{l}\text { Philosophers stone or hill (ancient magic } \\
\text { stone-shaphire). }\end{array}$ \\
\hline 67 & Exodus 48:18-Nepheq-sapphire. & & Ancient magic stone-shaphin. \\
\hline 68 & Esther 9:24 "Yi-Phila-Phur/Pur. Jonah 1:7 & & To cast lots, to find out, 1 Sam 14:38-42. \\
\hline 69 & $\begin{array}{l}\text { Philosapher (Niphilaosophru) Psalm 73:28, Judges } \\
\text { 6:13, Psalm 75:1, Niphulaosapher; Job 5:9 (9:10). }\end{array}$ & & $\begin{array}{l}\text { Ancient teachings, and of secret words } \\
\text { Numbers (inexplicable). }\end{array}$ \\
\hline 70 & Phalashaphat (Zephaniah 2:3) & & Judgment, Deut 17:7-11, Deut 19:15-19. \\
\hline 71 & $\begin{array}{l}\text { Zechariah 5:8, "Phiha” (mouth) of the Ephah: Zech } \\
\text { 5:5-11. Amos 8:5. }\end{array}$ & & Symbol of evil \\
\hline 72 & $\begin{array}{l}\text { Supha (BDB, 2005, p. 693), Isaiah 5:28, Hosea 8:7, } \\
\text { Isaiah 21:1 (swear in Psalm 83:16) Job 37:9 } \\
\text { (Exodus 23:5, the Nile). } \\
\text { Yum-suph = Red Sea }\end{array}$ & & $\begin{array}{l}\text { Storm wind, Ocean waves, Red Sea-surfing or } \\
\text { Rushing water }\end{array}$ \\
\hline 73 & $\begin{array}{l}\text { BDB, 2007, p. } 703 \text { "Saah is Supha (rush of storm } \\
\text { wind) Job 14:19. }\end{array}$ & & Heavy rain or ocean water. \\
\hline 74 & $\begin{array}{l}\text { Hebrew “Sopheret" is Scribe Teacher or writer (1 } \\
\text { Chro 2:55, Neh 7:57) Ezra 2:55 }\end{array}$ & & \\
\hline 75 & Heleph = Philo (to turn around) & & \\
\hline 76 & $\begin{array}{l}\text { Hebrew Sopher (i.e., learned man) } 1 \text { Chro 27:32 = } \\
\text { Ezra: Neh 8:1-9, Joshua 15:15-16, Judges 1:11-12 }\end{array}$ & & \\
\hline 77 & $\begin{array}{l}\text { Seloph: Proverbs 15:4 Proverbs 22:12, (21:12), Job } \\
\text { 12:19, Exodus 23:8; Seleph = the mighty. }\end{array}$ & & crookedness \\
\hline 78 & $\begin{array}{l}\text { Keryriath-sepher (Joshua 15:15-16) (Colossians 2:8 } \\
\text { philosepher = pagan or false teacher) }\end{array}$ & Didaskalos or Grammateas. & $\begin{array}{l}\text { City of scribes/teachers (Maer, } 1988 \text { on } \\
\text { Josephus), BDB, 2007, pp. 704-708. }\end{array}$ \\
\hline 79 & $\begin{array}{l}\text { Sarpha, Seapha, Soephim (1 Kings 18:21) (BDB, } \\
\text { 2007, p. 703) Isaiah 2:21, Isaiah 10:33, Ezekiel } \\
\text { 31:6 \& 8, Isaiah 27:6 }\end{array}$ & Sophist (crafty) & $\begin{array}{l}\text { Branches, shoot out, cleft, bough, branches of } \\
\text { trees or branches of opinions Psalm 119:113, } \\
\text { "Double or many-minded". }\end{array}$ \\
\hline
\end{tabular}


(Table 1 continued)

\begin{tabular}{|c|c|c|c|}
\hline & Hebrew & Greek & English \\
\hline 80 & $\begin{array}{l}\text { Baalseaphim = phallaseaphim = phelsoephim }(1 \\
\text { Kings } 18: 21) \text { Isaiah 2:6-following many opinions = } \\
\text { Pha or } \mathrm{Ba}=\text { paqu = pagan }\end{array}$ & & $\begin{array}{l}\text { Divided opinions, in-between (Isaiah 57:5; } \\
\text { polytheism). }\end{array}$ \\
\hline 81 & $\begin{array}{l}\text { Saphad (Zechariah 12:12) Phalas (to mourn, ponder } \\
\text { or reflect; to ruminate over or examine). }\end{array}$ & & To wail, mourn or reflect. \\
\hline 82 & $\begin{array}{l}\text { Saphah; Psalm 40:15 Losophothah = to snatch } \\
\text { (philosophot = He who captures, or misleads) Job } \\
\text { 34:32 }\end{array}$ & $\begin{array}{l}\text { Philosophia (to entice, snare, trap or } \\
\text { mislead) }\end{array}$ & $\begin{array}{l}\text { To catch, capture or snatch away (see } \\
\text { Colossians 2:8, Psalm 40:15). }\end{array}$ \\
\hline 83 & 1 Kings 18:21 “Baalsoephim” & Philosophy & Bad opinion, godless opinion. \\
\hline 84 & $\begin{array}{l}\text { Phalasaphim (magicians) or magic pebbles. Jonah } \\
\text { 1:7 }\end{array}$ & Psephia (Revelation 13:18) Psephon & $\begin{array}{l}\text { Psephon = figure out, untie a difficulty or } \\
\text { calculate, or choose by balloting or casting lots. }\end{array}$ \\
\hline 85 & Numbers 26:5-11 phallusphaha & Psephon & Rebel family (Isaiah 50:8 opposition) \\
\hline 86 & Psalm 119:113, Job 20:1 (or 22) “Seaphim”. & & $\begin{array}{l}\text { Double mindedness, unreliable. } \\
\text { To doubt religions. }\end{array}$ \\
\hline 87 & Tephilin (Mathew 23:1-5) & $\begin{array}{l}\text { Phylactery or phylasso or phylate } \\
\text { (to protect or protected by magic) } \\
\text { which gave us prophylactic in } \\
\text { modern medicine. }\end{array}$ & $\begin{array}{l}\text { Jewish law box tied to the head like protective } \\
\text { magic band. }\end{array}$ \\
\hline 88 & Hosea 7:1; phalasaqer (Phalasaphqar) & philosophia & To falsify-falsehood. \\
\hline 89 & $\begin{array}{l}\text { Ezekiel 13:8-20 “Casaph or Kisittot” is plural for } \\
\text { Qesemim, i.e., (sorcery). }\end{array}$ & Pharmakon & $\begin{array}{l}\text { Magic: to find out through magic or the } \\
\text { extraordinary called "Philu” (Jonah 1:1-7, } 1 \\
\text { Samuel 14:38-42, etc.) }\end{array}$ \\
\hline 90 & Daniel 8:21 “Sephere” (Zepher) & & Greece is a He-Goat, i.e., I took know. \\
\hline 91 & $\begin{array}{l}\text { Nehemiah 7:57, Ezra 8:55, “Sopherim” or } \\
\text { Sophereth” }\end{array}$ & & $\begin{array}{l}\text { Scribes, writers, ancient script copiers, teachers, } \\
\text { etc. }\end{array}$ \\
\hline 92 & $\begin{array}{l}\text { Joshua 15:15-16 kyriath-sepher Philal-Yoseph (I } \\
\text { thought or assumed; Gen 48-11 to Judges) }\end{array}$ & Grammatikos & $\begin{array}{l}\text { Sopher-teacher or scribe, Ezra 7:11-12, Neh } \\
8: 1-9\end{array}$ \\
\hline 93 & $\begin{array}{l}\text { Note: Kiriath-Sepher (c1, 450BC) c900 years } \\
\text { before Greek philosophy, BDB, 2007, p. } 951 .\end{array}$ & Didaskalos and Didactos & $\begin{array}{l}\text { City of scribes, writers and teachers (1 } \\
\text { Chronicles 2:55). }\end{array}$ \\
\hline 94 & $\begin{array}{l}\text { Rephe, Rephili (to back slide) Ruth 1:4 \& } 14 \\
\text { (Hebrew Orphah = Araphili) godless }\end{array}$ & $\begin{array}{l}\text { Greek “Aphiemi” (to figure or } \\
\text { withdraw). }\end{array}$ & To leave, withdraw, forgive, refrain or to drop. \\
\hline 95 & $\begin{array}{l}\text { Exodus 4:24-26, v } 26 \\
\text { Hebrew “Rephili” (to forgive or let go) } \\
\text { Hebrew "Rapha”-to rapair, to remove, or to heal }\end{array}$ & & $\begin{array}{l}\text { Colossians 2:8-13, philosophia referred to } \\
\text { Jewish teachers teaching that forgiveness comes } \\
\text { through circumcision (mul-rephil). }\end{array}$ \\
\hline 96 & $\begin{array}{l}\text { Genesis 18:23\&24, “Ha-aph-ti-sephet to carry } \\
\text { snatch or sweep away; Col 2:8) }\end{array}$ & & (BDB, 2014) Topheth Jer 7:32 big fire \\
\hline 97 & $\begin{array}{l}\text { Genesis } 19: 15 \text { \& } 17 \text { "Phen-ti-Sapha (Col 2:8) to } \\
\text { carry or sweep away (to destroy) }\end{array}$ & & To destroy \\
\hline 98 & $\begin{array}{l}\text { Hosea 6:5 Isaiah 29:13, Psalm 71:15, Zephaniah } \\
\text { 2:3, Psalm 50:16 “Phi-losepher-Phiq(Human) } \\
\text { teachings }\end{array}$ & & \\
\hline 99 & $\begin{array}{l}\text { Genesis 50:1 “Phioyoseph” (worthless human } \\
\text { wisdom) }\end{array}$ & & Worthlessly human \\
\hline 100 & $\begin{array}{l}\text { Deuteronomy 25:1-3 Phenosapha “(Human } \\
\text { additions Deut 4:2) }\end{array}$ & & \\
\hline 101 & $\begin{array}{l}\text { Gen 15:5-Losepher (Psalm 50:16) Losepher-Phil = } \\
\text { to count or teach. }\end{array}$ & & \\
\hline 102 & Zephanian is sophoniah (mystery) & & Hidden (BDB, 561) \\
\hline 103 & Ebal (Phal) shepho & & Not ordained by God \\
\hline 104 & Niphala sophu (Amos 5:2 = 2 Sam 3:34) = Col 2:8 & & To fall and die = outdated \\
\hline 105 & Philosipor (Amos 3:5) & & A trap cannot catch a bird \\
\hline 106 & $\begin{array}{l}\text { Ha-phlashaphya (Dan 1:20, 2:4 \& 27, Num 11:4 } \\
\text { (godless people) }\end{array}$ & & Magic and witchcrafts \\
\hline 107 & $\begin{array}{l}\text { Phentosophiq or Phenosophiq (Gen 19:15 \& 17: } \\
\text { 1Sam 15:6) }\end{array}$ & & To destroy (Psalm 40:4; Nephesy-Losophoteq) \\
\hline
\end{tabular}

\section{References}

Bible Society of Nigeria. (2014). Holy bible: Good news translation. Lagos: BSN.

Brown, A. P., \& Smith, B. W. (2008). A readers Hebrew Bible. Grand Rapids: Zondervan. 
Brown, F., Drivers, S., \& Briggs, C. (2014). The Brown-Driver-Briggs Hebrew and English lexicon (Biblical Aramaic). Massachusetts: Hendrickson.

Douglas, J. D. (1993). The New Greek English interlinear New Testament. (R. K. Brown, and P. W. Comfort, Eds.). Illinois; Tyndale House Publishers, Inc.

Eke, J. (2001). The Igbo - English Dictionary-Okowa Okwu. Enugu: New Generations.

Harris, L. R., Archer, G. L., \& Watke, B. K. (1980). Theological (Hebrew Old Testament) Wordbook. Chicago: Moody Publishers.

Hebblethwaite Brian. (1980). Problems of theology in theology and philosophy. Cambridge: Cambridge University Press.

Honderich, T. (1987). Philosophy through its past. Middlesex: Penguin, San Diego Academic.

International Bible Society. (1984). The New International Version (NIV) of the Holy Bible. Colorado Springs: IBS.

Kanachikuzhy, A. (2008/2013). The New Community Bible (NCB) Catholic Edition. Philippines, Pombeai and Ibadan st. Paul Publications.

Kittel, G., Friedrich, G., \& Bromiley, G. W. (1985). Theological dictionary of the (Greek) new testament. Grand Rapids: Eerdmans.

Mansoor Menachem. (2002). Step by step Hebrew. Illinois: Baker.

Metzger, B. (2001). The Greek new testament lexicon. Illinois: Baker.

Nestle Publications. (1971). Greek New Testament cited by Bruce Metger (2001) The Greek New Testament Lexicon. Illinois: Baker.

New, C. W., \& Phillips, C. E. (1953). World history: From ancient times to 1760. Toronto and Canada: Clark and Dent.

Newman, B., Aland, K., Black, M., Martini, C. M., Metzger, B. M., \& Wikgren, A. (1983). The Greek new testament and lexicon. New York: United Bible Societies.

Pfeiffer, C., \& Harrison, E. (1953). The Wycliffe Bible commentary: Collins goodnews translation of the Holy Bible. Chicago: Moody Publishers.

Robert, H. (1960). Great books of the Western world. Chicago: Britarnica.

Robinson, M., \& Davidson, G. (2007). Chambers 21st century dictionary. Edinburgh: Chambers.

Simpson E.J and Weiner E.S.C (1989) The Encyclopedic Dictionary of Current English (New York; Oxford) 